\title{
Phylogeny-wide conservation and change in developmental expression, cell-type specificity and functional domains of the transcriptional regulators of social amoebas
}

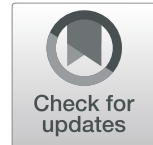

\author{
Gillian Forbes, Zhi-hui Chen, Koryu Kin, Hajara M. Lawal, Christina Schilde, Yoko Yamada and Pauline Schaap* (1)
}

\begin{abstract}
Background: Dictyostelid social amoebas self-organize into fruiting bodies, consisting of spores and up to four supporting cell types in the phenotypically most complex taxon group 4. High quality genomes and stage- and cell-type specific transcriptomes are available for representative species of each of the four taxon groups. To understand how evolution of gene regulation in Dictyostelia contributed to evolution of phenotypic complexity, we analysed conservation and change in abundance, functional domain architecture and developmental regulation of their transcription factors (TFs).
\end{abstract}

Results: We detected 440 sequence-specific TFs across 33 families, of which 68\% were upregulated in multicellular development and about half conserved throughout Dictyostelia. Prespore cells expressed two times more TFs than prestalk cells, but stalk cells expressed more TFs than spores, suggesting that gene expression events that define spores occur earlier than those that define stalk cells. Changes in TF developmental expression, but not in TF abundance or functional domains occurred more frequently between group 4 and groups 1-3, than between the more distant branches formed by groups $1+2$ and $3+4$.

Conclusions: Phenotypic innovation is correlated with changes in TF regulation, rather than functional domain- or TF acquisition. The function of only 34 TFs is known. Of 12 TFs essential for cell differentiation, 9 are expressed in the cell type for which they are required. The information acquired here on conserved cell type specifity of 120 additional TFs can effectively guide further functional analysis, while observed evolutionary change in TF developmental expression may highlight how genotypic change caused phenotypic innovation.

Keywords: Dictyostelia, Evolution of transcriptional regulation, Evolution of phenotype, Comparative genomics, Comparative transcriptomics, Amoebozoa

\section{Background}

Multicellularity enables organisms to specialize their cells for different functions and to organize the specialized cells into a wide array of tissues and organs. Cell-type specialization results from selective gene transcription, which is largely achieved by the binding of sequencespecific transcription factors upstream of the trancription start site in the $5^{\prime}$ intergenic regions of protein coding genes. The regulation of the activity of these factors by intercellular communication and environmental cues is one

* Correspondence: p.schaap@dundee.ac.uk

School of Life Sciences, University of Dundee, DD15EH, Dundee, UK of the major mechanisms that allow fertilized eggs to develop into functioning adults. The duplication and diversification transcription factor genes and their expression is considered to have been a major mechanism for acquisition of ever-increasing cell-type specialization and organismal complexity in the course of evolution [1].

Dictyostelid social amoebas represent an early type of multicellularity where cells feed as individuals, but come together when starved to form multicellular aggregates. The aggregates transform into migrating slugs and fruiting bodies, which, depending on the species, contain spores and up to four more cell-types [2]. This life cycle

(c) The Author(s). 2019 Open Access This article is distributed under the terms of the Creative Commons Attribution 4.0 International License (http://creativecommons.org/licenses/by/4.0/), which permits unrestricted use, distribution, and reproduction in any medium, provided you give appropriate credit to the original author(s) and the source, provide a link to the Creative Commons license, and indicate if changes were made. The Creative Commons Public Domain Dedication waiver (http://creativecommons.org/publicdomain/zero/1.0/) applies to the data made available in this article, unless otherwise stated. 
evolved from that of the solitary amoebas, which encyst individually when starved. Encystment still occurs in some Dictyostelia, when conditions for aggregation are unfavourable [3].

We aim to understand how the gene regulatory mechanisms that caused cell-type specialization evolved in early multicellular organisms, using the genetically tractable Dictyostelia to investigate this problem. Molecular phylogenies subdivide Dictyostelia into four major and some minor groups $[4,5]$, with most novel cell types appearing in group 4 [6, 7], which contains the model organism Dictyostelium discoideum. Following completion of the $D$. discoideum genome sequence [8], we obtained genome sequences for a representative species in each of the three other taxon groups, which were almost fully assembled by primer walking $[9,10]$. Others and ourselves obtained transcriptome data across taxon groups of purified cell types and during developmental progression into fruiting bodies and cysts, both earlier [10-12] and in this work. The high quality genomes and transcriptomes allow us to retrace changes in the abundance, expression profiles, cell type specificity and functional domain architecture of Dictyostelium transcriptional factors (TFs) throughout the course of their evolution.

We here present conservation and change in 440 sequence-specific and 42 general TFs of Dictyostelia, highlighting associations between particular TF families and specific developmental roles, taxon groupspecific gene amplification and loss, and evolutionary changes in the cell-type specificity and developmental regulation of TFs.

\section{Results}

\section{Identification and conservation of transcription factor} families

The genomes of $D$. discoideum (Ddis) and D. purpureum (Dpur) in group 4, D. lacteum (Dlac) in group 3, P. pallidum (Ppal) in group 2 and D. fasciculatum $(D f a s)$ in group 1 were screened for the presence of members of the 97 known eukaryotic families of sequence-specific transcription factors [13]. Groups 1, 2, 3 and 4 have recently been reclassified as families with the names Cavenderiaceae, Acytosteliaceae, Raperosteliaceae and Dictyosteliaceae, while Dlac, Ppal and Dfas have been renamed to Tieghemostelium lacteum, Heterostelium album and Cavenderia fasciculata [14]. However, this classification was based on the single gene small subunit ribosomal DNA phylogeny [4], which was superseded by more robust multi-gene phylogenies, which only partially support the new classification $[5,15]$. We therefore continue to use the older nomenclature here.
In the first round of screening, TFs were retrieved from species proteomes by the Interpro identifier for the functional domain that defines each TF. In the second round, BLASTp or tBLASTn searches were performed on local proteome or genome libraries using signature TF sequences as query. For apparently incomplete orthologous groups, additional BLAST queries were performed with one of the orthologs. Table 1 lists the TF families that were and were not detected in Dictyostelia, with the number of different family members for the former. In total we detected 440 different TF genes, subdivided into 33 families, with 4 families being first identified in Dictyostelia.

To understand orthology relationships between family members and map species-specific gene gain and loss, we inferred phylogenetic trees for each family. To assess whether TFs underwent functional change in the course of evolution, the proteins were annotated with their functional domain architectures, which also provided supporting evidence for the orthology of proteins that grouped together. This is for example evident for clades 1 and 4 of the E2F/DP winged helix TFs shown in Fig. 1. To assess whether TFs underwent changes in developmental expression and/or cell type specificity, we used published RNAseq data of Ddis and Dpur developmental time courses and purified prestalk and prespore cells [11], purified Ddis spore-, stalk-, cup- and vegetative cells [12], Dlac, Ppal and Dfas developmental time courses and Ppal purified spores and stalk cells [10], as well as unpublished time courses of Ppal encystation and Dlac purified spore, stalk and vegetative cells. All RNAseq data are comprehensively listed in Additional file 2: Table S1 Because the different species do not complete development at the same time, developmental stages rather than time points were compared between species. For example, Fig. 1 shows that the $e 2 f$ and $t f d p 2$ genes and their orthologs in group-representative species are all upregulated at aggregation and tend to be more highly expressed in prespore cells in Ddis and Dpur. Neither gene is consistently upregulated in either of the mature cell types, but the Ppal e2f ortholog shows some upregulation in encystation.

Similarly annotated phylogenetic trees for all other sequence-specific transcription factor families are shown in Additional file 1: Figure S1 - S16, accompanied by summary descriptions of known roles of the factors within and outwith Dictyostelia. We also searched for orthologs of the general transcription factors (gTFs), which make up and/or associate with the preinitiation complexes that are required for transcription of all genes (Additional file 1: Figure S17). The information on conservation of individual TF genes and their domains, developmental expression and cell-type specificity across Dictyostelia is listed per family in Additional file 3: Table S2 and schematically represented in Figs. 2 and 3 for families 
Table 1 Sequence-specific transcription factors detected in Dictyostelia

\begin{tabular}{|c|c|c|c|}
\hline \multicolumn{4}{|c|}{ Eukaryote sequence-specific transcription factor families } \\
\hline in Dictyostelia & $\mathrm{n}$ & not in Dictyostelia & \\
\hline AATF & 1 & AFT & MADF \\
\hline ARID/BRIGHT & 3 & Alfin-like & MATa1 \\
\hline AT hook & 47 & AP2/GBD/EREBP/ERF & MBD \\
\hline $\mathrm{bHLH}$ & 4 & AP-2/bHSH & mTERF \\
\hline bZIP & 23 & APSES/KilA-N & NAC/NAM \\
\hline $\mathrm{C} 2 \mathrm{H} 2 \_\mathrm{ZnF}$ & 103 & B3NP1/IAA /ARF & PAX \\
\hline C2HC5_ZnF & 1 & ABF1_ARS1 & PLATZ \\
\hline CBF/NF-Y & 12 & $\mathrm{BBR} / \mathrm{BPC}$ & POU \\
\hline Crtf & 2 & BES1/BZR1/LAT61 & Pros/Prox \\
\hline Cud & 6 & Brinker & Rap1 \\
\hline $\mathrm{CXC}$ & 1 & CENPB & RFX \\
\hline E2F/DP & 9 & CG-1/CAMTA & $\mathrm{RHR} / \mathrm{RHD}$ \\
\hline EnY2 & 1 & COE/EBF & Runt \\
\hline FAR1/FRS & 1 & Copper-fist & S1FA \\
\hline Gal4-like & 5 & Grainyhead/CP2/LSF & SAND/KDWK \\
\hline GATA & 65 & CSD & SART-1 \\
\hline$G B F$ & 6 & CSL/LAG1 & SBP/SQUAMOSA \\
\hline GCFC & 3 & CUT/ONECUT/CDP & Sigma70 \\
\hline HMG & 7 & DBP/DNC & SMAD/MH1 \\
\hline Hox & 30 & DM/Doublesex & STK/GeBP-like \\
\hline HSF & 1 & EIN3/EIL & T-box \\
\hline Jmj-C & 14 & Ets & TCP \\
\hline Lambda & 2 & LEAFY/LFY/FLO & TEA/ATTS/TEF \\
\hline MADS/SRF & 6 & FKH/Fox & THAP \\
\hline MIZ & 6 & GCM & Trihelix/GT \\
\hline Myb/SANT & 56 & GCR1 & VHR1 \\
\hline Ndt80/PhoG & 3 & GRAS & Whirly/PBF2 \\
\hline NF-X1_ZnF & 4 & GTF2I-like & zf-C2HC \\
\hline Psq & 9 & $\mathrm{HB}-\mathrm{PHD} / \mathrm{ZF}-\mathrm{HD}$ & zf-C4 \\
\hline STAT & 4 & $\mathrm{IBD}$ & zf-CXXC \\
\hline$T F 2$ & 1 & IRF & zf-Dof \\
\hline TMF-1 & 1 & LOB/LBD/AS2 & zf-HRT \\
\hline WRKY & 3 & & \\
\hline
\end{tabular}

Families of eukaryote sequence-specific TFs, retrieved from [13] that were detected in Dictyostelia contrasted to other eukaryote TF families not found in Dictyostelia. The number of different genes (n) detected across Ddis, Dpur, Dlac, Ppal and Dfas is indicated. Families in italics/bold are unique to Amoebozoa

with less or more than 50 members, respectively, and in Additional file 1: Figure S18 for the gTFs. For each recorded feature, we also calculated the distribution of the different states of that feature across the individual larger TF families (Figs. 4 and 5).
Overall, 35\% of sequence-specific and $86 \%$ of gTFs were conserved over all five genomes (Fig. 4a). The Dpur genome is most often missing an ortholog, but this is likely an artefact due to it being the only partially assembled draft genome. The large family of GATA TFs shows the most extensive genome-specific gain of individual members. Across sequence-specific TFs, gene amplification occurs about equally frequently in Ddis, Dpur and Ppal, but is lower in Dfas and much reduced in Dlac (Fig. 4b), which correlates with and may partially cause the small genome size of Dlac (23 Mbp versus 31-35 Mbp for the others $[9,10])$.

\section{Conservation of functional domains and developmental expression}

Functional domain architecture is conserved in the greater majority of orthologs (Fig. 5a), except for the AT-hook and $\mathrm{C} 2 \mathrm{H} 2 \mathrm{TFs}$, where the small domains (12 amino acids for AT-hook, 23 amino acids for C2H2) are often not recognized in some orthologs. Compared to a set of 385 developmentally essential genes [10], the domain architecture of TFs is mostly simple, containing little else than the signature DNA binding domain. There is therefore less opportunity for domain change. More than half of all orthologous sets of TFs show differences in the developmental expression profiles of their member genes. Because change in gene expression may cause individual TF's to take on novel roles, we were particularly interested in the phylogenetic distribution of such changes. Figure 5b shows that across TF families, developmental expression was most frequently divergent in only one species. In those cases where it was divergent in two or three species, the difference most frequently occurred between group 4 and the other groups and less frequently between the more distantly related branch I and branch II, or scattered across the phylogeny. This is particularly evident in the compiled sets of all sequencespecific TFs, the combined families with three or less members and the general TFs (1st, 2nd and last bars of Fig. $5 \mathrm{~b}$ ) and for the E2F_DP and MIZ TFs. On the other hand, for bZIPs divergent gene regulation occurred only scattered across the phylogeny.

Divergence in functional domain architecture also affects single species most, but is then mostly scattered across the phylogeny (Fig. 5a) and the same is true for conservation of the TF genes themselves (Fig. 4c). This difference between conservation of gene function and conservation of gene expression was also observed for the set of 385 developmentally essential genes, where changes in gene expression were more group 4-specific and changes in functional domains more scattered across the phylogeny [10]. Analysis of 25 phenotypic traits over 99 Dictyostelium species showed that the most dramatic changes in phenotype occurred in the last 


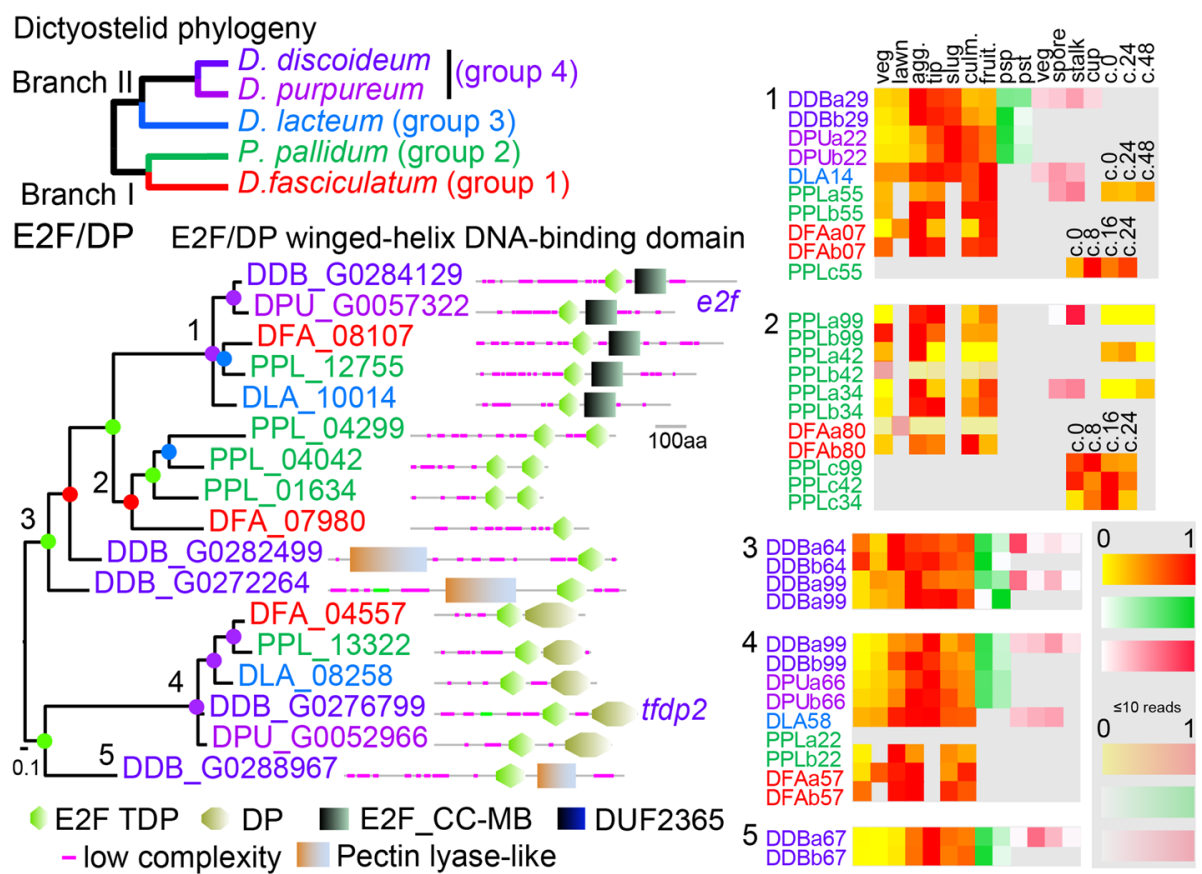

Fig. 1 Conservation and change in E2F/DP function and expression across Dictyostelia. Proteins containing E2F/DP winged helix DNA binding domains were identified by their Interpro identifier IPR003316 and BlastP search of five taxon-group representative dictyostelid proteomes. The sequences corresponding to the E2F/DP domains were aligned and a phylogeny was inferred by Bayesian analysis [16], and decorated with the functional domain architecture of the proteins analysed using SMART [17]. Locus tags and gene names are colour coded to reflect the taxon group of the host species, as shown in the dictyostelid phylogeny. Clades of orthologous genes or other groupings are annotated with relative transcript levels, shown as heat maps, at different developmental stages (yellow-red: 0-1 fraction of maximum value), prespore or prestalk cells (white-green: 0-1 fraction of summed reads), or vegetative, spore, stalk and cup cells (white-red: $0-1$ fraction of summed reads). Sets with maximally 10 or less reads are shown in wash-out color. The normalized transcript reads were retrieved from published [10-12] or novel RNA sequencing experiments and are all listed in Additional file 2: Table S1. Note that some developmental stages like "lawn" and "slug" are not represented in one or both Ppal and Dfas time courses. The transcript profiles are preceded by the first three and last two digits of the locus tags, while "a" and "b" represent replicate experiments, except for spore, stalk, cup and vegetative cells where the average of a triplicate experiment was used. Developmental stages: veg.: vegetative; lawn: starving cells, agg.:aggregation; tip: tipped mounds; slug:migrating slugs; culm.:early to mid fruiting bodies; fruit:: completed fruiting bodies, c.0 - c.48: hours into encystation

common ancestor to group $4[6,7]$. The current and earlier analyses of genotypic change indicate that these phenotypic innovations were more likely caused by changes in the regulation of existing genes than by the appearance of novel genes or novel functional domains. The observed limited importance of change in functional domains does however not exclude that more subtle mutations that alter gene function strongly affect phenotypic evolution.

When comparing developmental expression profiles across TF families (Fig. 5c), it is striking that except for the general transcription factors which are mostly constitutively expressed, over $70 \%$ of the sequencespecific transcription factors are upregulated after the transition from growth to development, with the small families of Cud and MIZ TFs being exclusively expressed in development. Early upregulation around the aggregate stage or a peak of expression in middevelopment are the most dominant expression profiles. Apart from the jmjC TFs, no sequence-specific
TFs are predominantly expressed in the vegetative stage.

\section{Cell-type specificity of transcription factors}

To investigate whether families of transcription factors are associated with specific cell fates, we also calculated how families with more than 3 members were percentage-wise expressed in each of the six scored cell types and for Ppal in the process of encystation. Across all sequence-specific TFs, 38\% was specifically expressed in the prespore cells and $18 \%$ in the prestalk cells of group 4 slugs, and this difference was even more extreme for the general TFs with 45 and 5\% expression in prespore and prestalk cells respectively (Fig. 5d). Only the JmjC and GATA families contained more members with prestalk than prespore expression, while no MADS or STAT TFs were specifically expressed in prespore cells and no E2F_DP, CBF or GBF TFs in prestalk cells. 


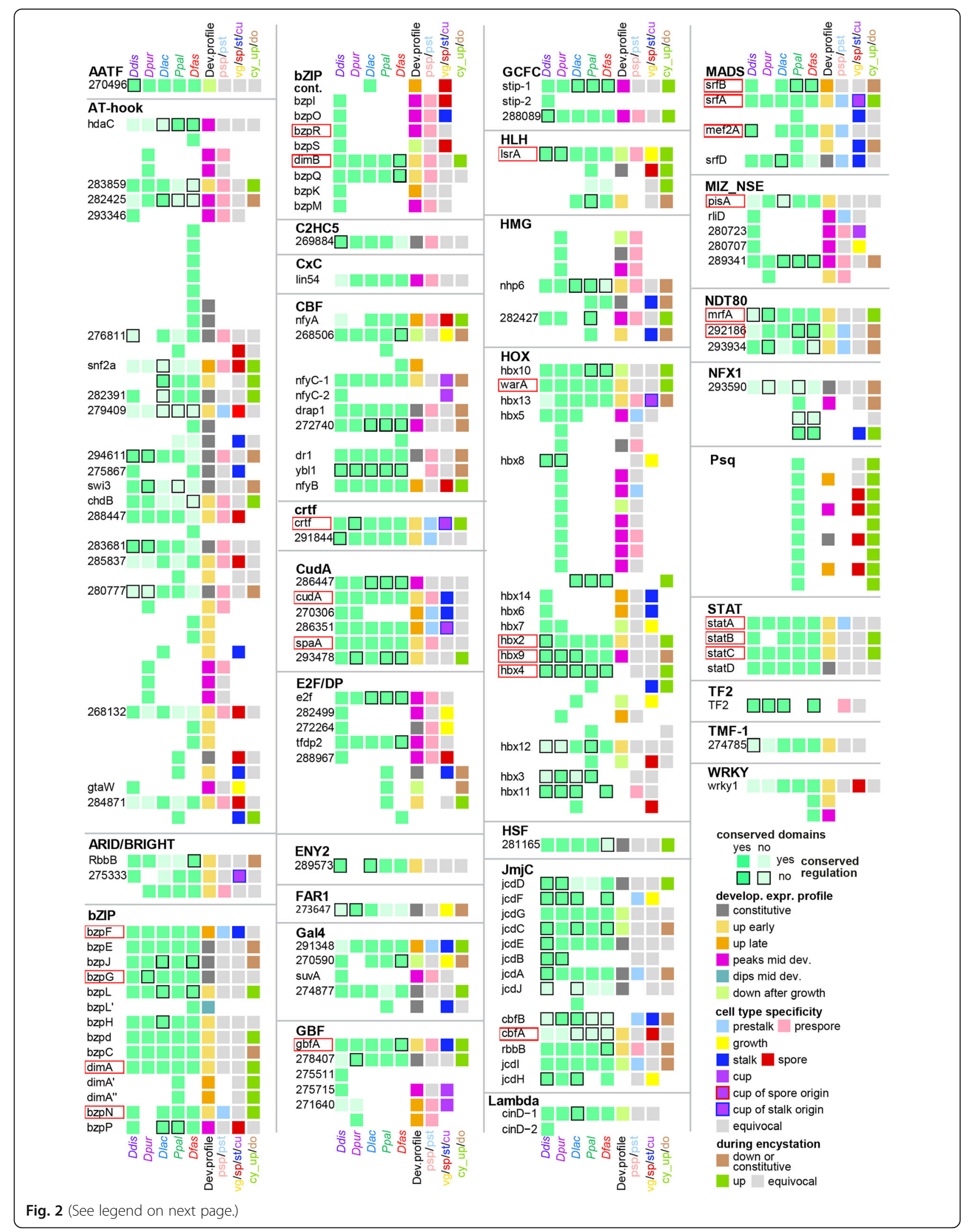


(See figure on previous page.)

Fig. 2 Phylogeny-wide change in sequence-specific TF families with $<50$ members. The presence of orthologous TF genes across the Ddis, Dpur, Dlac, Ppal and Dfas genomes is indicated by green squares below species names, which are shown in a lighter tone or with a black border, when compared to the majority, the functional domains or the developmental regulation, respectively, are not conserved. Where the number of nonconserved features is larger than 3, all differ from each other. The colour coding of the 6th, 7th and 8th square in each row respectively represent the developmental expression profile in the majority of species, the prestalk/prespore specificity when conserved between Ddis and Dpur slugs, the spore or stalk specificity when conserved between species, the cup and vegetative cell specificity in Ddis. The 9th square represents up- or down regulation in encystation of Ppal. Cup cells are only present in group 4 and are bordered red or blue when the orthologs in group 2 or 3 show spore- or stalk-specific expression, respectively. Grey reflects lack of specificity or conflicting data between species or replicate experiments and white reflects absence of data. The genes are listed by the Ddis gene names or 12 digit Dictybase gene identifiers from which the DDB_G0 prefix was omitted. The names of genes with known biological roles in Ddis are bordered in red. The gene identifiers and locus tags for the Dpur, Dlac, Ppal and Dfas genes are listed in Additional file 1: Table S2 together with all data on which this figure and Fig. 3 and Additional file 1: Figure $\mathrm{S} 18$ are based

In the fruiting body stage, this cell fate specificity was almost reversed for the sequence-specific TFs, of which $14 \%$ were expressed in spores and $17 \%$ in stalk cells (Fig. 5e). Another 5\% of TFs were expressed in cup cells, a population that is derived from prestalk cells $[12,18,19]$. This suggests that most genes that define the spore phenotype are already expressed in the slug stage, but that those that define the stalk and cup phenotypes are only expressed late in fruiting body formation. Here there was also evidence for more celltype preference of TF families, with bZIP and AT-hook TFs favouring expression in spores and the GATAs, Hox TFs and members of the small families of Gal4, MADS and Cud TFs favouring expression in stalk cells. CBFs, GBFs and MIZ TFs favour expression in cup cells. For the MADS TFs, their stalk and cup preference is consistent with their prestalk preference, but for the GBFs it is the reverse of their prespore preference.

As was also evident from the developmental profiles (Fig. 5c), many more sequence-specific TFs are specifically expressed during development into fruiting bodies than in the vegetative stage, but this not the case for the general TFs, which as expected are more constitutively expressed. Finally, in Ppal, where in addition to multicellular development, starving amoebas can also individually encyst, over $30 \%$ of members of all families are upregulated during the encystation process.

\section{Predicted roles for TFs from cell-type specificity and developmental profiles}

Information on stage- and cell-type specificity provides a clue on the possible developmental role of individual TFs and we therefore subdivided individual transcription factors into sets according to the cell-type and stage at which they are expressed. The sets with different celltype specificity are listed in Table 2 and sets sorted with respect to similar developmental stage of expression or different combinations of stage- and cell type specificity are listed in Additional file 4: Table S3. For an overview that combines data on TF expression in mature (MCT) and presumptive (PCT) cell types and stage of expression, we subdivided all cell type specific TFs into subsets according to their developmental expression profile and presumptive or mature cell fate. Figure 6 shows that prepore-specific TFs mostly show peak expression in mid development or are upregulated early, while out of 113 prespore-specific TFs, only 14 are also spore-specific and 9 become stalk-specific. The number of prestalk-specific TFs is at 52 less than half that of the prespore TFs and most prestalk TFs are upregulated early. 14 prestalk TFs are also stalk-specific, while 3 become spore-specific. Of the 17 cup-specific TFs, 4 were enriched in prestalk cells and 3 in prespore cells. Of the 91 TFs that are upregulated in Ppal cysts, 50 are also upregulated in multicellular development. 19 cystupregulated TFs are also expressed in mature spores and 9 in stalk cells. Like cysts, spores and stalk cells are surrounded by cellulosic walls. Apparently encystation shares many TFs with multicellular development, with both processes adapting cells to starvation and their metabolism towards cell wall biosynthesis.

Lastly, we explored the extent to which cell type specificity predicts TF function. Of the $254 \mathrm{TFs}$ detected in Ddis, there is only functional information from gene knock-outs and knock-down studies for 34 TF genes. Deletion of 12 TFs causes specific defects in, or lack of, terminally differentiated cell types and 9 of these TFs are only expressed in the cell type that is lost upon knockout (Additional file 5: Table S4). Deletion of 9 TFs causes alterations in the proportion of prespore and prestalk cells. Of this set only 2 TFs are specific to the diminished cell-type and 1 TF is specific to the increased cell type. The remaining 6 TFs are not cell-type enriched. This suggest that cell-type specificity of TFs predicts their role in ultimate cell fate well, but that cell type proportioning is subject to more subtle crossregulation. Also, logically, a TF that instigates a presumptive cell fate has to be present before that fate is assigned.

\section{Discussion}

Across five genomes that represent the four major groups of Dictyostelia, around 440 different sequence- 


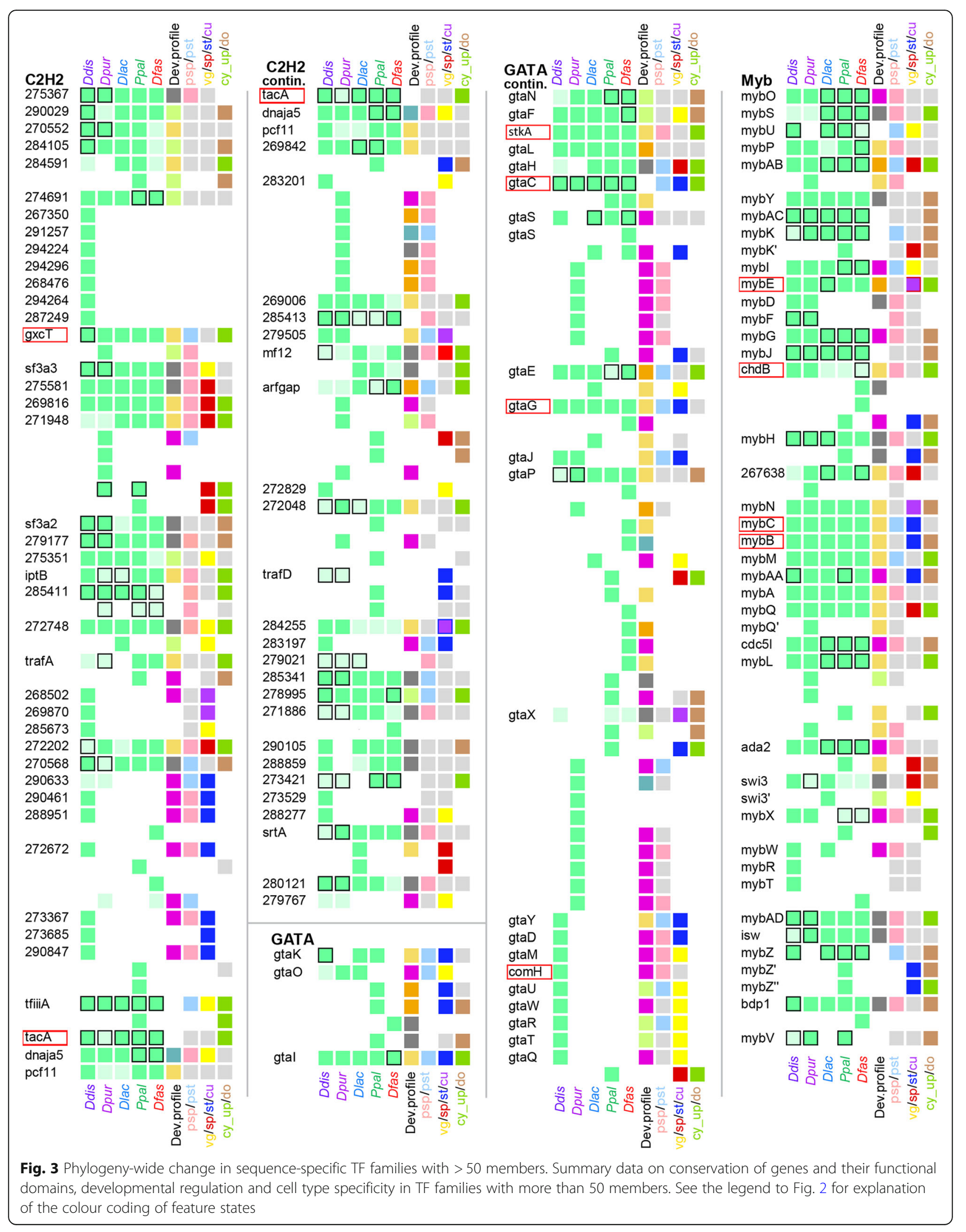




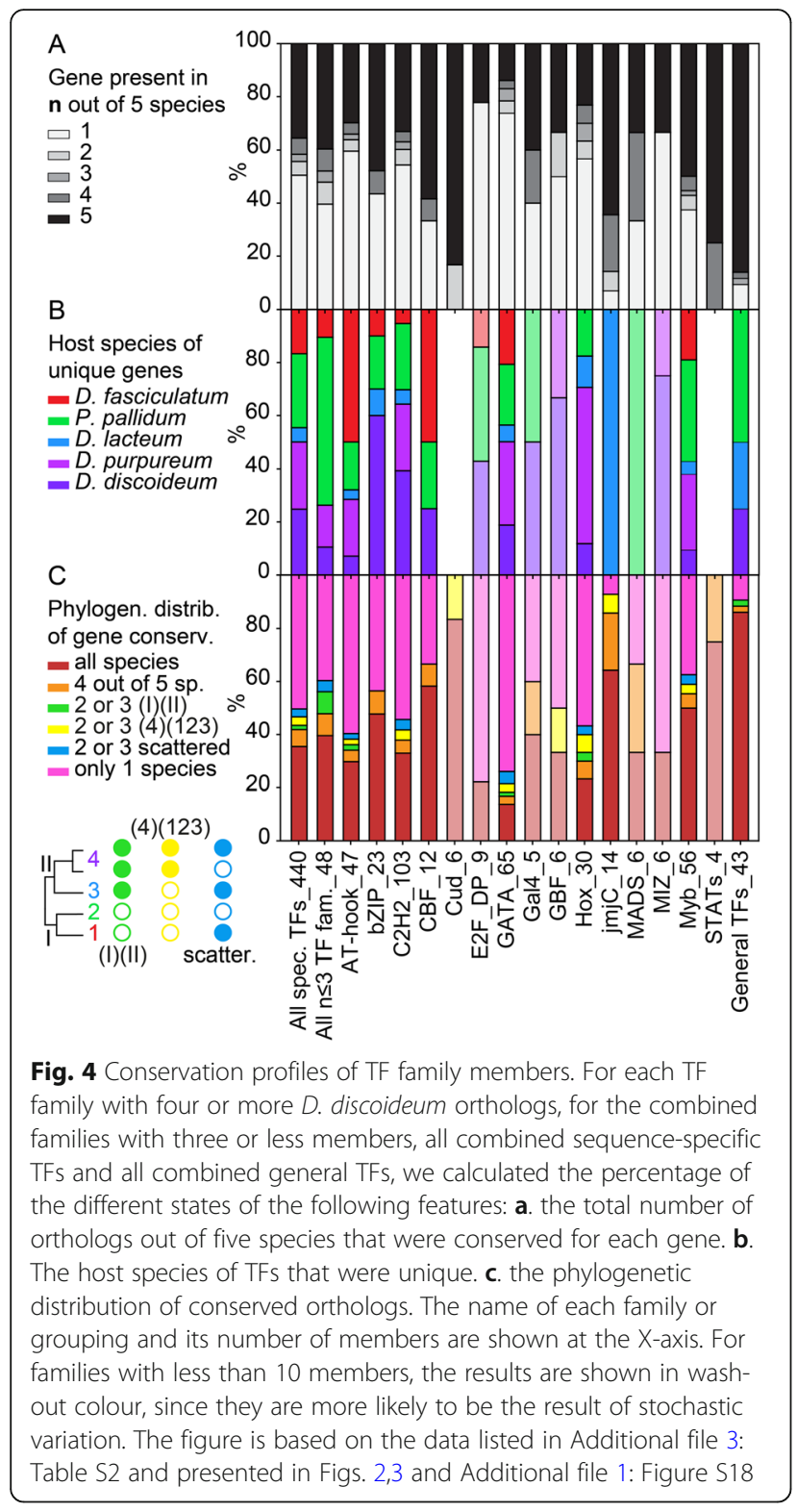

specific TFs across 33 TF families were detected. Due to genome- and species-specific gene amplification, this is about twice the number of TFs present in individual genomes. For instance, we detected 254 TFs in Ddis (as opposed to 106 in the initial genome annotation [8]), of which a core set of $181 \mathrm{TFs}$ is conserved across at least three other genomes.

The large family of GATA TFs is subject to extensive single gene amplification and the number of conserved genes in this family is therefore low. On the other hand, members of the almost equally large family of Myb TFs are mostly conserved. Nine members of the Pipsqueak family are unique to one genome (Ppal) and are all strongly upregulated in encystation. Gene amplification occurred about equally across four genomes, but was much lower in the Dlac genome, which is also $1 / 3 \mathrm{rd}$ smaller than the other four.

Changes in developmental expression profiles of conserved TFs occurred more frequently between group 4 and groups 1-3, than between the more distantly related branches I and II. This correlates with phenotypic change, which is also most pronounced between group 4 and the other three groups $[6,7]$. Since group 4 has neither more novel TFs nor more different functional domains in its TFs, this suggests that altered expression of existing TFs plays an important role in phenotypic innovation.

There are marked differences between TF families in developmental expression with e.g. $78 \%$ of bZIPs being developmentally up-regulated and $77 \%$ of jmjC TFs being constitutively expressed or developmentally downregulated. Not surprisingly, most (65\%) of the general TFs are constitutively expressed or down-regulated after growth, but across all sequence-specific TFs, $68 \%$ are developmentally up-regulated. This suggests that most of the Dictyostelid sequence-specific transcriptional machinery serves the developmental programme, with a relatively low number of TFs left to adapt cells to environmental challenges in the growth stage.

The prespore cells in slugs express over two times more TFs than the prestalk cells, with particularly many AT-hook, CBF, E2F-DP, GBF and general TFs being prespore-specific. However, this changes in the fruiting body stage, when the stalk cells express somewhat more TFs, with some smaller families like the CudA-like, Gal4-like, GbfA-like and MADS TFs being solely expressed in cells of the stalk and cup. Strikingly, TFs that are essential for spore formation, such a cudA, spaA and stkA [20-22] are expressed in prespore, but not spore cells, as if upon sporulation their task is finished. This pattern is similar across all prespore-specifc TFs, of which only $12 \%$ persists into the spores. For the prestalk-specific TFs, 34\% remain expressed in the stalk and cup. This temporal disparity in cell type specific gene expression likely reflects the different ontogenies of the mature cell types. The prespore cells start prefabrication of the spore wall in Golgi-derived vesicles after aggregation. The vesicles fuse with the plasmamembrane during spore maturation, thus rapidly completing the cell wall [23]. In contrast, stalk cells start cell wall synthesis gradually from the tip at the onset of fruiting body formation, while most cup genes are only expressed once the fruiting body is fully formed [12].

About 34 of the 254 TF genes of Ddis have been deleted, resulting in specific loss of or severely defective mature cell types for 12 TFs. For 9 out of 12 cases, the TF was in normal development expressed in the affected cell type and all $12 \mathrm{TFs}$ were conserved throughout Dictyostelia. This implies that bioinformatics-based 


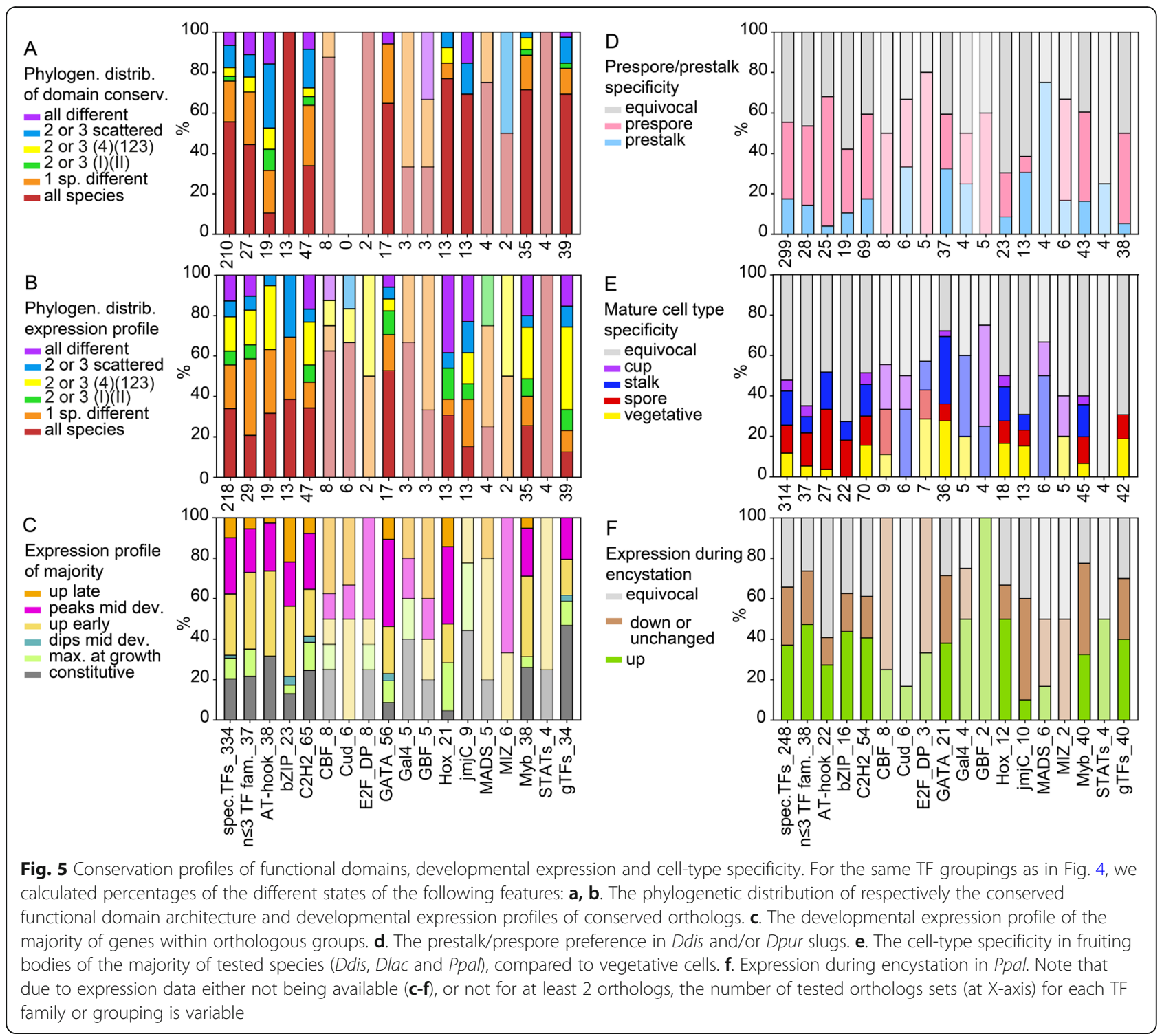

evidence on cell-type specificity and gene conservation is likely a useful tool for guiding discovery of the function of many of the remaining $220 \mathrm{TF}$ genes.

\section{Conclusions}

Dictyostelia jointly contain 440 different sequencespecific TFs, which are subdivided across 33 families, of which four are thus far unique to Amoebozoa.

Only $32 \%$ of sequence-specific TFs are expressed constitutively or during growth, while the rest is developmentally up-regulated, indicating that most of transcriptional machinery serves the multicellular phase of the life cycle.

Changes in developmental expression of TFs, but not in TF functional domains or TF gene gain or loss, are correlated with major changes in phenotype across
Dictyostelia, suggesting that altered expression of TFs is a major driver of phenotypic change.

The study presents detailed information on cell-type specificity of TFs, which correlates with an essential role in cell differentiation for 9 out of 12 TFs with known functions. This makes the current analysis an effective tool for gene function discovery.

\section{Methods}

\section{Sequence retrieval and phylogeny reconstruction}

TF protein sequences were firstly retrieved from the Ddis, Dlac, Ppal and Dfas genomes using the Interpro (https://www.ebi.ac.uk/interpro/) domain identifiers of all known TF families as query in the "advanced search" option of the social amoeba comparative genome browser SACGB (http://sacgb.fli-leibniz.de/cgi/index.pl). For Dpur a similar query was performed in the Pubmed 
Table 2 Cell-type specific transcription factors

\begin{tabular}{|c|c|c|c|c|c|c|c|c|c|}
\hline \multicolumn{2}{|c|}{ Prespore-specific } & \multicolumn{2}{|c|}{ Prespore-contin. } & \multicolumn{2}{|c|}{ Spore-specific } & \multicolumn{2}{|c|}{ Prestalk-specific } & \multicolumn{2}{|c|}{ Stalk-specific } \\
\hline Family & Gene & Family & Gene & Family & Gene & Family & Gene & Family & Gene \\
\hline \multirow[t]{12}{*}{ AThook } & 283859 & Cud & cudA & AThook & snf2a & AThook & 279409 & AThook & 275867 \\
\hline & 282425 & & spaA & & 279409 & bZIP & bzpF & bZIP & bzpF \\
\hline & 293346 & $\mathrm{CxC}$ & $\operatorname{lin} 54$ & & 288447 & & bzpN & & bzpO \\
\hline & 276811 & E2DP & e2f & & 285837 & $\mathrm{C} 2 \mathrm{H} 2$ & 270568 & $\mathrm{C} 2 \mathrm{H} 2$ & 290633 \\
\hline & snf2a & & 272264 & & 268132 & & 290633 & & 290461 \\
\hline & 294611 & & tfdp2 & & 284871 & & 279505 & & 288951 \\
\hline & 288447 & & 288967 & bZIP & bzpP & & 283197 & & 272672 \\
\hline & 283681 & Gal4 & suvA & & bzpl & & 285341 & & 273367 \\
\hline & 285837 & GATA & stkA & & bzpS & & 278995 & & 273685 \\
\hline & 280777 & & gtaD & $\mathrm{C} 2 \mathrm{H} 2$ & 275581 & Crtf & crtf & & 290847 \\
\hline & 268132 & & gtaM & & 269816 & & 291844 & & trafD \\
\hline & 284871 & & $\mathrm{comH}$ & & 271948 & Cud & 270306 & & 283197 \\
\hline \multirow[t]{6}{*}{ bZIP } & bzpl & GBF & gbfA & & 272202 & & 286351 & CudA & cudA \\
\hline & bzpO & & 271640 & & mf12 & Gal4 & 291348 & & 270306 \\
\hline & bzpR & GCGF & 288089 & $\mathrm{CBF}$ & nfyA & GATA & gtak & Gal4 & 291348 \\
\hline & $\operatorname{dim} B$ & HLH & IsrA & & nfyB & & gtaO & GATA & gtak \\
\hline & bzpQ & HMG & nhp6 & E2DP & 288967 & & gtal & & gtal \\
\hline & bzpM & & 282427 & GATA & gtaH & & gtaH & & gtaC \\
\hline \multirow[t]{22}{*}{$\mathrm{C} 2 \mathrm{H} 2$} & 275367 & Hox & $\mathrm{hb} \times 13$ & Hox & hbx11 & & gtaC & & gtaG \\
\hline & sf3a3 & & hbx5 & JmjC & cbfA & & gtaE & & gtal \\
\hline & 275581 & & $\mathrm{hbx} 11$ & myb & mybAB & & gtaG & & gtaY \\
\hline & 269816 & JmjC & $\mathrm{rbbB}$ & & mybK & & gtal & & gtaD \\
\hline & 271948 & MIZ & 280723 & & 267638 & & gtaY & GBF & gbfA \\
\hline & 279177 & & 289341 & & mybQ & & gtaU & Hox & hbx14 \\
\hline & 280121 & myb & mybo & & swi3 & & gtaR & & hbx6 \\
\hline & iptB & & mybs & WRKY & wrky1 & Hox & hbx5 & JmjC & $\mathrm{cbfB}$ \\
\hline & 285411 & & mybP & Cup-spe & & JmjC & $\mathrm{jcdF}$ & MADS & srfD \\
\hline & 272748 & & mybD & Family & Gene & & $j c d A$ & myb & mybH \\
\hline & 272202 & & mybF & ARID & 275333 & & $\mathrm{cbfB}$ & & mybC \\
\hline & 290461 & & mybG & $\mathrm{C} 2 \mathrm{H} 2$ & 268502 & & jcdl & & mybB \\
\hline & 288951 & & chdB & & 269870 & MADS & srfA & & mybAA \\
\hline & 272672 & & mybH & & 279505 & & mef2A & & mybz \\
\hline & 273367 & & 267638 & & 284255 & & srfD & & \\
\hline & 290847 & & mybA & $\mathrm{CBF}$ & nfyc-1 & MIZ & rliD & & \\
\hline & dnaja5 & & cdc5l & & nfyc-2 & Myb & mybu & & \\
\hline & 285413 & & ada2 & Crtf & crtf & & mybAB & & \\
\hline & $\mathrm{mf12}$ & & mybX & Cud & 275333 & & mybK & & \\
\hline & 271886 & & mybW & GATA & 268502 & & mybl & & \\
\hline & srtA & & mybAD & GBF & 269870 & & mybC & & \\
\hline & 280121 & & isw & & 279505 & & mybM & & \\
\hline $\mathrm{C} 2 \mathrm{HC} 5$ & 269884 & & bdp1 & Hox & 284255 & & mybz & & \\
\hline \multirow[t]{2}{*}{ CBF } & nfyA & TF2 & $\mathrm{tf} 2$ & MADS & srfA & NDT80 & 292186 & & \\
\hline & drap1 & & & MIZ & 280723 & & 293934 & & \\
\hline
\end{tabular}


Table 2 Cell-type specific transcription factors (Continued)

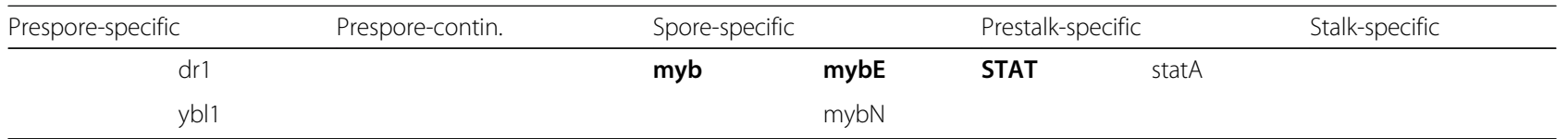

All TFs that are specifically expressed in the prestalk or prespore cells of slugs or in the spore, stalk and cup cells of fruiting bodies in the majority of species are listed. The second column contains either the gene name for annotated genes or the Dictybase 12 digit gene identifier minus the DDB_G0 prefix. Gene names/IDs are shown in bold when the biological role of the gene in Ddis is known

"protein" option (https://www.ncbi.nlm.nih.gov/pubmed) with the combined query "Dictyostelium purpureum and [Interpro domain identifier]". Next, a BLAST library was prepared in CLC-workbench v8.0 (https://www.qiagenbioinformatics.com) from the combined Ddis, Dpur, Dlac, Ppal and Dfas proteomes, downloaded from Dictybase (http://dictybase.org/) and SACGB, which was queried with the protein sequences of representative functional domains of each TF family.

The domain architectures all detected proteins were analysed using SMART [17], with the visual display of the architecture saved as an .svg file. The domain coordinates were used to isolate the sequences corresponding to the TF functional domains. These sequences were subsequently aligned using Clustal Omega [24] with 5 combined iterations. When functional domain sequences were short, a stretch of 20 amino-acids flanking the domain on either side was included in the alignment. Phylogenies were constructed using RAxML in Topali v2.5 [25] or MrBayes v3.2.6 [16], with the latter run for $10^{6}$ generations, using a mixed amino acid model with rate variation between sites estimated by a gamma distribution. When otherwise conserved genes appeared to be absent from species, their proteomes or genomes were queried once more by BLASTp or tBLASTn, respectively, using the orthologous sequence as bait. Phylogenetic trees were then reconstructed, including the novel sequences. Trees were rooted at midpoint using FigTree v1.3.1. and saved as .svg files. The tree .svg file was combined with the domain architecture .svg files for each protein in Adobe Illustrator CS5.

\section{RNA sequencing and analysis}

To obtain total RNA for Dlac stalk, spore and vegetative cells, amoebas were co-cultured with Klebsiella aerogenes on lactose-peptone agar. For vegetative cells, cells were harvested before bacteria started to clear. For stalk and spore cells, cells were harvested, freed from bacteria and incubated for $24 \mathrm{~h}$ on non-nutrient agar until fruiting bodies had formed. Spores were separated from stalks and RNA was isolated from the three cell types as described previously [12]. The qualities of the RNAs isolated in three independent experiments were assessed with TapeStation (Agilent) to be good (RIN > 7.5) and cDNA libraries were prepared using the Truseq Stranded mRNA Library Prep Kit (Illumina) with Low Sample Protocol. 75-bp paired end reads were sequenced with Illumina NextSeq 500

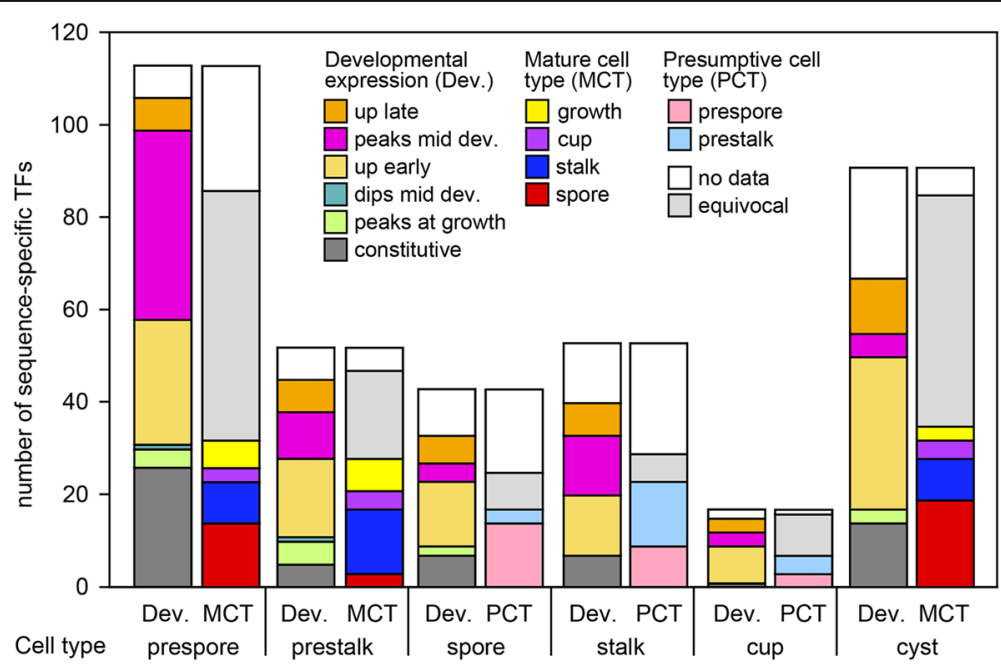

Fig. 6 Expression subsets of cell-type specific transcription factors. The sets of prestalk, prespore, spore, stalk, cup and cyst-specific TFs were subdivided into subsets according to the developmental stages at which they were expressed (Dev. bars). Prespore, prestalk and cysts TFs were subdivided in sets according to the mature cell types - spore, stalk, cup, feeding - in which they were expressed (MCT bars), while spore, stalk and cup-specific TFs were subdivided into sets according to expression in prestalk and prespore cells (PCT bars). The total numbers of TFs in each subset are shown. The identities of all TFs in the subsets are listed in Additional file 4: Table S3 
at the Tayside Centre for Genomic Analysis in two independent runs. The qualities of the RNA-Seq reads were inspected with FastQC [26]. The RNA-Seq reads were then mapped to the previously assembled transcriptome of D. lacteum [27] using RSEM [28] with the bowtie 2 aligner and with the read start position distribution (RSPD) estimation option. The read counts were normalized to Transcripts Per Million (TPM) [29] with RSEM.

To monitor gene expression during Ppal encystation, Ppal PN500 was co-cultured with $K$. aerogenes on LP agar. Cells were freed from bacteria and incubated at $2.5 \times 10^{6}$ cells $/ \mathrm{ml}$ in $250 \mathrm{mM}$ sorbitol in $20 \mathrm{mM} \mathrm{K}$-phosphate to induce encystation [30]. Total RNA was extracted with an RNAeasy Midi Kit (Qiagen), directly after harvest $(\mathrm{t}=0 \mathrm{~h})$ and after 8,16 and $24 \mathrm{~h}$ of incubation at $22^{\circ} \mathrm{C}$, at which point $80 \%$ of cells had encysted. Library construction, sequencing and sequence quality control and mapping of transcripts to the Ppal genome [9] were performed by Eurofins Genomics (https://www. eurofinsgenomics.eu/). Paired-end Illumina sequencing was performed on the Hi-seq2000 platform using the TruSeq (TM) SBS v5 sequencing kit. A total of 177,292, 620 reads containing $8.8 \mathrm{Mb}$ were obtained. The reads were mapped to the Ppal genome, using BWA 0.5.8c software (http://bio-bwa.sourceforge.net). The read counts were then normalized to reads per kilobase per million mapped reads (RPKM).

\section{Comparative transcriptomics}

For comparative analysis of developmental expression and cell type specificity of TF genes across the Dictyostelid phylogeny, normalized read counts from published and purpose-sequenced gene expression studies were combined into a single spreadsheet (Additional file 2: Table S1). The data include i. replicate developmental profiles for Ddis and Dpur obtained by Illumina sequencing, combined with RNAseq data of purified prestalk and prespore cells of migrating slugs [11], ii. Averaged read counts of three RNAseq experiments comparing purified spore-, stalk- and cup cells from mature Ddis fruiting bodies and vegetative cells [12], iii. Averaged read counts of three RNAseq experiments comparing purified spore- and stalk cup cells from Dlac fruiting bodies and vegetative cells. iv. A single developmental profile for Dlac and replicate developmental profiles for Ppal and Dfas [10], combined for Ppal with RNAseq data of purified stalk and spore cells and 24 and $48 \mathrm{~h}$ time points of encystation, vi. A separate $24 \mathrm{~h}$ time course of Ppal encystation. The developmental profiles are aligned between species with respect to developmental stage, rather than developmental time because species do not develop at the same rate. For each set of orthologous genes, or groups of amplified genes, the normalized read counts for each of the features listed above were transferred to Excel files and recalculated as fraction of the maximum read count for developmental profiles and as fraction of the sum of counts for cell-type specificity data. The conditional formatting option in Excel was used to generate heat maps, which were matched up with the phylogenetic trees in Adobe Illustrator.

\section{Supplementary information}

Supplementary information accompanies this paper at https://doi.org/10. 1186/s12864-019-6239-3.

\section{Additional file 1: Figure S1-S18. Annotated phylogenetic trees of} transcription factor families.

Additional file 2: Table S1. Gene expression profiles. Normalized transcript read counts of developmental time series and purified cell types from five Dictyostelium species.

Additional file 3: Table S2. Transcription factor conservation. Conservation and change in the presence, developmental expression and functional domain architecture in transcription factors across five Dictyostelid genomes.

Additional file 4: Table S3. Transcription factors grouped by cell-type and stage specificity. The data of Additional file 3: Table S2 were compiled and sorted to generate sets that were expressed in the same cell type or at the same developmental stage, or that shared a combination of the same stage and cell type specificity.

Additional file 5: Table S4. Transcription factors with known knock-out phenotypes. Knock-out phenotypes of transcription factors combined with their cell-type- and stage specificity of expression.

\section{Abbreviations}

BLAST: Basic Local Alignment Search Tool; Ddis: Dictyostelium discoideum; Dfas: Dictyostelium fasciculatum; Dlac: Dictyostelium lacteum; Dpur: Dictyostelium purpureum; Ppal: Polyspondylium pallidum; SMART: Simple Modular Architecture Research Tool; TF: Transcription factor

\section{Acknowledgements}

Not applicable.

\section{Authors' contributions}

GF performed most of the bioinformatics and wrote parts of the manuscript. PS designed the study and completed the manuscript. ZC, HL, CS and YY analysed some TF families. CS isolated RNAs during an encystation time course and KK isolated cell-type specific RNAs and performed RNAseq data analysis. All authors have read and approved the manuscript

\section{Funding}

YY and PS were supported by Wellcome Trust grant 100293/Z/12/Z, GF, HL and CS were supported by ERC grant 742288, ZC was supported by Leverhulme Trust grant RPG-2016-220 and KK was supported by an EMBO Long-term fellowship and Marie Curie Action ALTF 295-2015 and by JSPS Overseas Research Fellowship H28-1002. The funding bodies played no role in the design of the study and collection, analysis, and interpretation of data or in writing the manuscript.

\section{Availability of data and materials}

The raw RNA-Seq data of Dlac cell types and Ppal encystation time series have been submitted to Arrayexpress https://www.ebi.ac.uk/arrayexpress/experiments/E-MTAB-7824 under accession number E-MTAB-7824. All other data generated or analysed during this study are included in the published article and its supplementary information files.

Ethics approval and consent to participate Not applicable. 


\section{Consent for publication}

Not applicable.

\section{Competing interests}

The authors declare that they have no competing interests.

Received: 17 June 2019 Accepted: 29 October 2019

Published online: 21 November 2019

\section{References}

1. Johnson AD. The rewiring of transcription circuits in evolution. Curr Opin Genet Dev. 2017;47:121-7.

2. Loomis WF. Cell signaling during development of Dictyostelium. Dev Biol. 2014;391:1-16

3. Du Q, Kawabe Y, Schilde C, Chen ZH, Schaap P. The evolution of aggregative multicellularity and cell-cell communication in the Dictyostelia. J Mol Biol. 2015;427:3722-33.

4. Schaap P, Winckler T, Nelson M, Alvarez-Curto E, Elgie B, Hagiwara H, Cavender J, Milano-Curto A, Rozen DE, Dingermann T, et al. Molecular phylogeny and evolution of morphology in the social amoebas. Science. 2006;314:661-3.

5. Singh R, Schilde C, Schaap P. A core phylogeny of Dictyostelia inferred from genomes representative of the eight major and minor taxonomic divisions of the group. BMC Evol Biol. 2016;16:251.

6. Romeralo M, Skiba A, Gonzalez-Voyer A, Schilde C, Lawal H, Kedziora S, Cavender JC, Glockner G, Urushihara H, Schaap P. Analysis of phenotypic evolution in Dictyostelia highlights developmental plasticity as a likely consequence of colonial multicellularity. Proc Biol Sci. 2013;280:20130976.

7. Schilde C, Skiba A, Schaap P. Evolutionary reconstruction of pattern formation in 98 Dictyostelium species reveals that cell-type specialization by lateral inhibition is a derived trait. EvoDevo. 2014:5:34.

8. Eichinger L, Pachebat JA, Glockner G, Rajandream MA, Sucgang R, Berriman M, Song J, Olsen R, Szafranski K, Xu Q, et al. The genome of the social amoeba Dictyostelium discoideum. Nature. 2005;435:43-57.

9. Heidel A, Lawal H, Felder M, Schilde C, Helps N, Tunggal B, Rivero F, John U, Schleicher M, Eichinger L, et al. Phylogeny-wide analysis of social amoeba genomes highlights ancient origins for complex intercellular communication. Genome Res. 2011;21:1882-91.

10. Gloeckner G, Lawal HM, Felder M, Singh R, Singer G, Weijer CJ, Schaap P. The multicellularity genes of dictyostelid social amoebas. Nat Commun. 2016:7:12085.

11. Parikh A, Miranda ER, Katoh-Kurasawa M, Fuller D, Rot G, Zagar L, Curk $T$, Sucgang $R$, Chen R, Zupan B, et al. Conserved developmental transcriptomes in evolutionarily divergent species. Genome Biol. 2010; $11: R 35$.

12. Kin K, Forbes G, Cassidy A, Schaap P. Cell-type specific RNA-Seq reveals novel roles and regulatory programs for terminally differentiated Dictyostelium cells. BMC Genomics. 2018:19:764.

13. Hughes TR. A handbook of transcription factors. Dordrecht: Springer; 2011

14. Sheikh S, Thulin M, Cavender JC, Escalante R, Kawakami SI, Lado C, Landolt JC, Nanjundiah V, Queller DC, Strassmann JE, et al. A new classification of the Dictyostelids. Protist. 2018:169:1-28.

15. Schilde C, Lawal HM, Kin K, Shibano-Hayakawa I, Inouye K, Schaap P. A well supported multi gene phylogeny of 52 dictyostelia. Mol Phylogenet Evol. 2019;134;66-73.

16. Ronquist F, Huelsenbeck JP. MrBayes 3: Bayesian phylogenetic inference under mixed models. Bioinformatics. 2003;19:1572-4.

17. Schultz J, Milpetz F, Bork P, Ponting CP. SMART, a simple modular architecture research tool: identification of signaling domains. Proc Nat Acad Sci U S A. 1998;95:5857-64

18. Sternfeld J, David CN. Fate and regulation of anterior-like cells in Dictyostelium slugs. DevBiol. 1982;93:111-8.

19. Yamada Y, Kay RR, Bloomfield G, Ross S, Ivens A, Williams JG. A new Dictyostelium prestalk cell sub-type. Dev Biol. 2010;339:390-7.

20. Fukuzawa M, Hopper N, Williams J. cudA: a Dictyostelium gene with pleiotropic effects on cellular differentiation and slug behaviour. Development. 1997;124:2719-28.

21. Chang W-T, Newell PC, Gross JD. Identification of the cell fate gene stalky in Dictyostelium. Cell. 1996;87:471-81.

22. Yamada $Y$, Cassidy A, Schaap P. The transcription factor spores absent a is a PKA dependent inducer of Dictyostelium sporulation. Sci Rep. 2018;8:6643.
23. West CM. Comparative analysis of spore coat formation, structure, and function in Dictyostelium. Int Rev Cytol. 2003:222:237-93.

24. Sievers F, Higgins DG. Clustal omega, accurate alignment of very large numbers of sequences. Methods Mol Biol. 2014;1079:105-16.

25. Milne I, Lindner D, Bayer M, Husmeier D, McGuire G, Marshall DF, Wright F. TOPALi v2: a rich graphical interface for evolutionary analyses of multiple alignments on HPC clusters and multi-core desktops. Bioinformatics. 2009; 25:126-7.

26. FastQC, a quality control tool for high throughput sequence data [https:// www.bioinformatics.babraham.ac.uk/projects/fastqc/].

27. Singh R, Lawal HM, Schilde C, Glockner G, Barton GJ, Schaap P, Cole C. Improved annotation with de novo transcriptome assembly in four social amoeba species. BMC Genomics. 2017;18:120.

28. Li B, Dewey CN. RSEM: accurate transcript quantification from RNA-Seq data with or without a reference genome. BMC Bioinformatics. 2011;12:323.

29. Wagner GP, Kin K, Lynch VJ. Measurement of mRNA abundance using RNAseq data: RPKM measure is inconsistent among samples. Theory Biosci. 2012;131:281-5.

30. Ritchie AV, van Es S, Fouquet C, Schaap P. From drought sensing to developmental control: evolution of cyclic AMP signaling in social amoebas. Mol Biol Evol. 2008;25:2109-18.

\section{Publisher's Note}

Springer Nature remains neutral with regard to jurisdictional claims in published maps and institutional affiliations.
Ready to submit your research? Choose BMC and benefit from:

- fast, convenient online submission

- thorough peer review by experienced researchers in your field

- rapid publication on acceptance

- support for research data, including large and complex data types

- gold Open Access which fosters wider collaboration and increased citations

- maximum visibility for your research: over $100 \mathrm{M}$ website views per year

At $\mathrm{BMC}$, research is always in progress.

Learn more biomedcentral.com/submissions 